http://jmscr.igmpublication.org/home/ ISSN (e)-2347-176x ISSN (p) 2455-0450

crossref DOI: https://dx.doi.org/10.18535/jmscr/v8i7.41

Journal Of Medical Science And Clinical Research

\title{
Edaravone: A Free Radical Scavenger with Multiple Pleotropic Actions can be a Potential Game Changer Agent in Prevention and Alleviation of COVID-19 - Induced Cytokine Storm
}

Authors

\section{Dr Butungeshwar Pradhan ${ }^{1 *}$, Dr Gourav Pradhan², Deblina Pradhan ${ }^{3}$}

${ }^{1}$ Associate Professor, Department of internal medicine. Institution:-VSSIMSAR, Burla, Sambalpur, Odisha, India.768017

${ }^{2,3}$ VSSIMSAR, Burla, Sambalpur, Odisha

*Corresponding Author

Dr Butungeshwar Pradhan

\begin{abstract}
Reactive oxygen species (ROS) and reactive nitrogen species (RNS) are produced in the cells through the mitochondrial respiratory chain during aerobic metabolism and in hypoxic conditions. Aged peoples have co-morbid diseases associated with depletion of endogenous anti-oxidants are in oxidative state, hyperresponsive lung with raised levels of IL-6.COVID-19 infection of the respiratory tract increases activation of pulmonary epithelial, endothelial cells and macrophages causes overproduction of cytokines, chemokines and free radicals induce massive sequestration of neutrophils within the pulmonary microvasculature transmigrate into alveolar space and release varieties of cytotoxic, pro-inflammatory compounds and free radicals prodigiously that perpetuates a vicious circle by recruiting additional inflammatory cells. Overproduction ROS causes depletion of anti-oxidants leading to oxidative stress and induces bystander injury in the host. Any critical illness associated with overproduction of ROS, which act as second messenger to activate the nuclear factor kappa- $\beta(N F-k \beta)$ and transcription factor activator protein-1(APC-1) cause aberrant production of many cytokines (IL-2,IL-1,IL-6 and TNF- $\alpha)$, hematopoietic growth factors and adhesion molecules. Cytokines such as $I L-2, I L-1, I L-6$ and TNF $\alpha$ have binding sites for $N F k \beta$ and APC-1 further perpetuating cytokine production in the vicious circle leading to cytokine storm with loss of immune regulation. Thus, ROS can regulate the production of cytokines. Edaravone as a broad spectrum anti-oxidant scavenges both water soluble and lipid soluble ROS have multiple pleotropic effects such as anti-inflammatory, immunomodulatory, anti-cytokine, anti-apoptotic, anti-necrotic, anti-fibrotic, membrane stabilizing, lung protective effects appears to be potentially useful agent in the prevention and therapy of COVID-19 induced cytokine storm and ARDS.
\end{abstract}

Keywords: COVID-19, Edaravone, Cytokine storm, ARDS, Antioxidant, Free radicals, ROS.

\section{Introduction}

Normal physiological process, such as cellular respiration generate small amount of oxidizing reactive oxygen species (ROS) and reactive nitrogen species (RNS). These free radicals play crucial role in activation of signalling pathways in animal and plant cells. These are chemically highly reactive molecules that can damage cell structures. However, there are endogenous defences (scavenger) or antioxidant systems to protect tissues from ROS and 
RNS induced injury. These antioxidants can be divided into enzymatic and non-enzymatic groups. The enzymatic antioxidants include superoxide dismutase (SOD), catalase and glutathione peroxidase. Their cofactors are selenium, zinc, copper, and manganese for SOD and iron for catalase. The non-enzymatic antioxidants include vitamin $\mathrm{E}, \mathrm{C}, \mathrm{A}$ or $\beta$-carotene etc. Vitamin $\mathrm{E}$ can directly quench ROS including $\mathrm{O}_{2}^{-}, \mathrm{HO}^{-}$and ${ }^{1} \mathrm{O}_{2}$. Vitamin $\mathrm{C}$ in higher doses broadly scavenges water soluble ROS including the major neutrophils oxidants $\mathrm{HO}^{-}, \mathrm{H}_{2} \mathrm{O}_{2}$ and hypochlorous acid. Vitamin $\mathrm{C}$ also is a pro-oxidant when combined with iron. ${ }^{1}$ Most of ROS are produced in cell through the mitochondrial respiratory chain reaction during endogenous aerobic metabolism, while in hypoxic situation generates nitric oxide (NO), which can produce other RNS. Free radicals have odd number of electrons, this make them short lived, highly reactive. Consequently it reacts quickly with other closest stable molecules/substances 'stealing' its required electron to obtain stability. Meanwhile, the attacked molecule become a free radical by losing its electron and start a chain reaction cascade causing damage to the living cells. ${ }^{2,3}$ The enzymatic and non-enzymatic antioxidants system are intimately interlinked to one another. Both vitamin $\mathrm{C}$ and glutathione implicated in recycling of $\alpha$ tocopherol. The complex interactions of these different antioxidants systems with narrow spectrum of activity may imply that successful therapeutic strategies will depend on the use of a combination of various antioxidants rather than a single agent. ${ }^{4,5}$

\section{Redox/oxidation status in the lung:}

The redox environment in the lung lining fluid is important factor in determining the lung's innate and adaptive immune system. Normal lung have extremely high levels of extracellular antioxidants function to maintain extracellular space in a highly reduced state and facilitate the maintenance of immune response. Balance between antioxidants and oxidants are sufficient in normal lung. In oxidized states there is presence of hyperresponsive immune system. Increase in oxidants or decrease in antioxidants can disrupt this balance, referred to as "oxidative state" and associated with diverse lung pathologies and facilitate the binding of pathogens or antigens to effectors cells leading to a hyperresponsive innate immune system leading to enhanced release of oxidants such as superoxide and NO causing activation of nuclear factor-kappa $\beta$ $(\mathrm{NK}-\mathrm{k} \beta)$ and enhance production of cytokines, including TNF- $\alpha$, interleukin-1 $\beta$, (IL-1 $\beta$ ), IL-12. Creation of markedly reduced environment by addition of antioxidants blunts all of the above primary response of innate immune system. ${ }^{6}$

\section{Redox/oxidation status in critical illness:}

Oxidative stress has been implicated in the manifestation of critical illness, including ischemia and reperfusion injury and systemic inflammatory states. Excessive ROS production may leads to indiscriminate bystander injury in the host. ROS causes direct cellular injury to cellular proteins and nucleic acids and by inducing lipid peroxidation, leading to destruction of the cell membrane. ${ }^{4}$ ROS also play a role as second messenger in the intracellular signalling pathways of inflammatory cells. In particular, the activation of the NF-k $\beta$ induced by hydrogen peroxide .NF-k $\beta$ resides in the cytoplasm as an inactive complex bound to its inhibitor I-k $\beta$. Upon stimulation with various agents including cytokines, viruses, ROS, mitogens causes dissociation of NF-k $\beta$ - Ik- $\beta$ complex and translocation of NF-k $\beta$ to the nucleus with its high affinity of binding to specific sites in the promoter region of target genes stimulating their transcription. $^{7} \mathrm{NF}-\mathrm{k} \beta$ is involved in the regulation of numerous pro-inflammatory genes, including many cytokines (TNF- $\alpha$, IL-1,IL-6,IL-8,IL-2), hematopoietic growth factors such as granulocytemacrophage-colony stimulating factor(GMCSF), cell adhesion molecules-1(CAM-1) i.e. intercellular adhesion molecule-1 (ICAM-1), endothelialleukocyte adhesion molecule-1(ELAM-1),vascular CAM-1(VCAM-1), and nitric oxide syntheses (i NO). A second messenger transcription factor activator -1(APC-1), which also seems to be regulated by changes in redox states of the cell may contribute to the ongoing inflammatory cytokine 
production and progression of systemic inflammation leading to end organs injury, manifested by the development of ALI/ARDS or multiple organ failure syndrome. In addition ischemia/reperfusion can leads to significant production of ROS by the increased activity of xanthine oxidase and increased production of hypoxanthine due to reintroduction of both. ${ }^{6}$

\section{Lungs in Systemic Inflammatory Response Syndrome (SIRS):}

Any initiating severe events (sepsis, shock, trauma, acute pancreatitis ect.) can leads to activation of acute inflammatory response on a systemic level. One of the earliest manifestations is activation of pulmonary endothelium and macrophages (alveolar and interstitial), upregulation of adhesion molecules and production of cytokines and chemokines that induce a massive sequestration of neutrophils within pulmonary microvasculature. These cells transmigrate across all endothelium and epithelium into the alveolar space and release varieties of cytokines and pro-inflammatory compounds including proteolytic enzymes, ROS, nitrogen species, cationic proteins, lipid mediators and additional inflammatory cytokines. This perpetuates a vicious circle by recruiting additional inflammatory cells that in turn produce more cytotoxic mediators leading to profound injury to the alveolo-capillary membrane leading to respiratory failure i.e. ALI/ARDS. The source of ROS/RNS in this context also includes itinerant and resident leukocytes (neutrophils, monocytes, and macrophages), parenchymal cells (endothelial and epithelial cells, fibroblast) and circulating oxidants generating enzyme xanthine oxidase. Leukocytes expressed two enzyme systems - (i) the NADPH oxidase, (ii) Nitric oxide syntheses (NOS) that generate substantial amount of ROS. Finally inhaled gas oxidant like high oxygen concentration used for mechanical ventilation causes ROS production. ${ }^{8}$

\section{Immunoscenesence:}

Age associated dysregulation of immune response contribute to higher incidence of infectious diseases in the elderly people due to imbalance of $\mathrm{Th}_{1} / \mathrm{Th}_{2}$ cytokine production and increased production of pro-inflammatory cytokines i.e. IL-6. Syntheses of many cytokines are implicated by changes in the cellular oxidant/antioxidant balance. Influenza A virus infection can cause early increase in IL-1, IL-6, and TNF- $\alpha$, GMCSF, interferon-y(IFN-y).But IL-6 remains elevated throughout the infection. The alveolar macrophage plays a prominent role in the initiation of an immune response during early stage of infection. The lung epithelial cells and peripheral blood mononuclear cells (PBMCs) increases production of IFN- $\alpha$. In elderly $\mathrm{Th}_{1}$ activity is decreased with decreased production of IL-2 and IFN- $\alpha$, while $\mathrm{Th}_{2}$ activity is increased with increased production of IL-6,IL-1 $\beta$, TNF- $\alpha$ and IL1Ra. The persistent elevated levels of IL- 6 may indicate presence of age-associated diseases like hypertension, diabetes, CVA, CAD, heart failure, COPD, CKD etc. ${ }^{9}$ Antioxidants such as vitamin E, $\mathrm{C}, \quad \beta$-carotene and glutathione enhance IFN production. Glutathione supplementation maintains intracellular redox balance; cellular defence against oxidative stress significantly increases IL-2 production and decrease TNF- $\alpha$. Vitamin E supplementation increases IL-2 production and decreases IL-6 in animal and humans. ${ }^{10}$

\section{Antioxidant regulates the production of cytokines:}

There are two possible mechanism, first through effects on transcription factor NF-k $\beta$ and APC-1, that regulated by redox status. Many cytokines (IL-2, IL-1, IL-6 and TNF- $\alpha$ ) contain NF-k $\beta$ and APC-1 binding sites in the promoter and enhancer region of the genes encoding them. Reduction /oxidation can either up or down regulate DNA binding and transactivation activities (or both) in transcriptional activator-dependent and cell type dependent manner. Vitamin $\mathrm{E}$ or its derivatives inhibit NF-k $\beta$ in human Jurkat $\mathrm{T}$ cells and also by other antioxidants differently in different cells. Thus, antioxidants have immunomodulatory role. ${ }^{\mathbf{1 1}}$ The second possible mechanism is through $\mathrm{PGE}_{2}$ synthesis: $\mathrm{PGE}_{2}$ inhibit early stage of $\mathrm{T}$ cell activation and decreases IL-2 production, modulates $\mathrm{Th}_{1} / \mathrm{Th}_{2}$ cytokine secretion through its effects on IL-12, which increases $\mathrm{Th}_{2}$ response promoting differentiation of naïve $\mathrm{T}$ cell 
into a population of $\mathrm{Th}_{1}$ cells capable of production of large amount of IFN-y. Vitamin E inhibits cyclooxygenase activity and decreases $\mathrm{PGE}_{2}$ production in age associated increased $\mathrm{PGE}_{2}$ synthesis and COX activity in old mice. ${ }^{\mathbf{2}}$ ROS are also generated by phagocytes following viral infection as the virus itself can change redox status of cells involved in generation of ROS directly and decreases total concentration of antioxidants such as glutathione, vitamin $\mathrm{C}$ and $\mathrm{E}$ from the lung in the early stage of infection attributed to increased production of oxidants. Antioxidant vitamin $\mathrm{E}$ supplementation in high doses increases NKactivity ( 3 fold) and decreases viral loads and lower pulmonary IL-6 and TNF- $\alpha$ levels and increase production of $\mathrm{Th}_{1}$ cytokines IL-2 and IFN-y in old mice. $^{13}$

\section{Pathogenesis of COVID-19-induced cytokine storm and ALI/ARDS:}

After coronavirus get inhaled and infects the respiratory epithelial cells through its ACE2 receptor, dendrite cell phagocytes the virus infected cells and present the antigens to $\mathrm{T}$ cells. The effectors $\mathrm{T}$ cells function by killing the infected cells and cytotoxic $\mathrm{CD}_{8}{ }^{+} \mathrm{T}$ cells produce and release pro-inflammatory cytokines which induces cell apoptosis. Both pathogens and infected cell apoptosis trigger and amplify immune response. There is exacerbation of cytokine production and excessive recruitment of immune cells into the lung and the uncontrollable epithelial cell damage generates a vicious circle for infection related to ALI/ARDS. ${ }^{14}$ In the early stage of viral infection dendrite cells and epithelial cells are activated and express a cluster of pro-inflammatory cytokines including IL- $1 \beta$,IL-2,IL-6 INF- $\alpha / \beta$, TNF- $\alpha$, and C$\mathrm{C}$ motif chemokines (CCL-3,CCL-5,CCL-2) and interferon inducible protein-10(IP-10) etc. Overproduction of these cytokines, chemokines and ROS contribute to the development of disease complications. Some patients have high levels of IL-10 secreted by the helper $\mathrm{Th}_{2}$ cells a marker of counter anti-inflammatory response associated with down regulation of neutrophils and macrophages function referred as "immune paralysis" and there is repressed immune response with hypoalbuminemia, lymphopenia, neutropenia and decreased percentage of $\mathrm{CD}_{8}{ }^{+} \mathrm{T}$ cells indicating immune suppression and patients may die after cytokine storm. The amplification of the inflammatory response promote cellular apoptosis or necrosis of the affected cells, which further fuel inflammation, followed by increase permeability of blood vessels and aberrant accumulation of inflammatory monocytes, macrophages and neutrophils in the lung's alveoli. This vicious circle intensifies the situation as the regulation of immune response is lost and cytokine storm is further activated resulting dire consequences. Thus, there is excessive inflammation with depressed immune system and activated cytokine storm substantially contribute to the pathogenesis of COVID-19 infection. ${ }^{\mathbf{1 5}}$ There also high levels of circulating serum ferritin in cytokine storm may reflect as an acute phase response and play a critical role in inflammation and ROS generation. ${ }^{16}$ Ferritin a intracellular iron storage constituting two subunit $\mathrm{H}$ and $\mathrm{L}$ and their ratio may differ depends on tissue type and physiologic status of the cell. $\mathrm{H}$ ferritin seems to be immunomodulatory function and have proinflammatory activity with induction of expression of different inflammatory mediators including IL$1 \beta .{ }^{17}$ Serum ferritin levels correlates with disease severity in COVID-19 infection. $^{18}$ Once ferritin released it loses part of the inner iron content giving rise to extremely high serum levels of 'free iron' and the excess free iron induce a marked procoagulalant state able to favour the production of hydroxyl radicals. Oxidative stress on RBCs and fibrin can induce production of dense clots responsible for stroke development. Iron chelating agent can taper the inflammatory response through a reduction of ROS production. ${ }^{19}$

COVID-19 infection induce cytokine storm have raised levels of Angiotensin-II (Ang-II) associated with oxidative stress and correlated to severity of infection:

As the ACE2 is primary receptor for COVID-19 infection, ACE2 expression is reduced leading to increased Ang-II levels and AT1R activation by 
Ang-II leading to hypertension, hypertrophy, fibrosis and oxidative stress causing lung injury. Whereas Mas receptor (MasR) activation by Ang-17 (product of ACE2 cleaving of Ang-II) results in effects opposite of AT1R activation i.e. vasodilatation, growth inhibition, antioxidant and anti-fibrotic actions and prevent lung injury. Reduced activity of ACE2 within the cell internalization and degradation of ACE2 are inhibited by AT1R blockers (ARBs) Losartan and ACEI have protective against lung injury. ${ }^{20}$

\section{$\mathrm{NAD}^{+}$Depletion:}

Both viral infection and RAAS activation produce ROS in a reproductive manner resulting to oxidative burst. Increased ROS levels have destructive effects on cellular macromolecules such as lipids, proteins especially nucleic acids. Oxidative stress mediated DNA damage is repaired primarily via base excision repair (BER) pathway. Normally poly-ADP-ribose polymerase-1 (PARP-1), a DNA base excision repair enzyme activated by DNA breaks and contribute to BER pathway for maintenance of genome stability. Upon activation of PARP-1, rapidly uses the substrate $\mathrm{NAD}^{+}$to transfer polyADP-ribose (PAR) to itself and nuclear receptor proteins and damaged DNA. PARP-1 has ADPribose transferase activity and function as an antiviral agent through ADP-ribosylation of viral genome (RNA) and inhibition of viral transcription translation. Excessive activation of PARP-1 occurs to compensate ADP-ribose hydrolyzation of viral macrodomain (NSP3) poly (ADP-ribose) glycohydrolase (PARG) which is associated with catalytic consumption of $\mathrm{NAD}^{+}$followed by ATP reduction leading to depletion of energy and cell death. $^{21}$

Endothelial dysfunction due to reduction of Nitric Oxide (NO) Production:-

Decreased NO production is prominent in COVID19 infection as NO degradation caused by ROS. The reduced NO bioavailability results in proliferative, pro-oxidant, pro-inflammatory and pro-thrombotic response. In hypoxic situation ROS generation and HIF-1 $\alpha$ activation occurs, which consequently induce expression of furin enzyme and viral activation and invasion to other non-ACE2 expressing cells. Decreased NO attributed to inflammation and endothelial dysfunction, induction of vascular smooth muscle cells (VSMCs) proliferation, LDL oxidation and vascular cell adhesion molecule-1 and monocyte chemoattractant protei-1(MCP-1) expression. $^{21}$

\section{Edaravone}

In 2001, edaravone was approved in Japan to treat acute-phase cerebral infarction. In 2017, the U.S.FDA approved for treatment of amyotrophic lateral sclerosis (ALS). Edaravone a member of substituted 2-pyrazolin-5-one class has the chemical name 3-methyl-1-phenyl-2-pyrazolin-5-one. In India available as clear colourless solution in glass ampoule containing $30 \mathrm{mg} / 20 \mathrm{ml}$ of water for injection, given IV infusion diluted with $100 \mathrm{ml}$ of isotonic saline in 30 minutes, twice daily for treatment of cerebral ischemic stroke.

\section{Pharmacokinetics and Metabolism:}

The maximum plasma concentration (Cmax) of edaravone at the end of IV infusion during clinical trials was a trend of a greater than dose proportional increase in concentration of area under the curve (AUC) and Cmax of edaravone, but no plasma drug accumulation was observed with multiple dose administration. The drug is $92 \%$ protein bound primarily to albumin and in the range of 0.1 to 50 micromole/L yields no concentration dependence. The mean terminal half-lives of edaravone and its metabolites were 4.5-6 and 2.0-2.8 hours respectively. The drug is metabolize to inactive sulfate and glucoronide conjugated in the liver and kidney. It is excreted mainly in the urine as its glucoronide conjugate form after $70 \%$ to $90 \%$ of the dose was recovered in the urine as sulfate conjugate. $^{22}$ In water at $\mathrm{pH} 7.4$ the percentage of the neutral and anionic forms of edaravone have been calculated to be $28.5 \%$ and $71.5 \%$ respectively Approximately a half of edaravone exists as anionic form at physiological $\mathrm{pH}$ is the active form of edaravone. As pKa of edaravone is 7.0 edaravone able to enter lipid environment such as cell membrane and scavenge peroxyl radicals generated from lipids in radical chain reactions. (Oxidation of 
lipids proceeds as a chain reaction in which a single radical can generate thousands of molecules of lipid hydroperoxide causing damage to biological membrane). Edaravone scavenges both lipid and water soluble peroxyl radicals exerts broad spectrum antioxidant activity acting in concert with $\alpha$-tochopherol and other antioxidant present in the body. $^{23}$

\section{Effective doses and blood levels in treatment of stroke:}

The plasma concentration of edaravone after its infusion at doses of 1.5 or $3.0 \mathrm{mg} / \mathrm{kg}$ body weight in rats cerebral ischemic model were 5.7 and $9.9 \mu \mathrm{M} / \mathrm{L}$. The effective concentration in vitro studies were between 1 and $10 \mu \mathrm{M} / \mathrm{L}$ and had no detrimental effects. Thus, $3 \mathrm{mg} / \mathrm{kg}$ IV dose of edaravone either bolus or infusion in 30 minute is effective therapeutic dose in cerebral ischemia and other diseases. ${ }^{24}$ Doses adjustment not required in patients with renal or mild to moderate hepatic impairment. No drug interaction known and not inhibit cytochrome p-450 enzyme, CYP2A2, CYP3B6 and CYP3A4. ${ }^{23}$

\section{Anti-inflammatory effects in strokes:}

Microglial cells are the resident immune cells mediate neuroinflammation in degenerative CNS diseases and strokes, activated to engage in different functions such as phagocytising the toxic cellular debris, producing pro-inflammatory cytokines and enhancing neuronal survival by release of tropic factors. Activation of microglial cells release excessive pro-inflammatory cytokines or cytotoxic factors such as NO, TNF- $\alpha$, IL-1 $\beta$ and ROS. Edaravone reduces infarct size, improve neurological scores by decreasing ROS generation, inhibiting production of pro-inflammatory mediators (TNF- $\alpha$, IL-1 $\beta$, NO, ROS) and prevent oxidative stress damage. Edaravone drastically decreases the number of activated microglial cells and suppress the upregulation of Toll- like receptor2 (TLR2) expression in microglial cells in acute strokes. ${ }^{25}$ The neuroprotective effects of edaravone includes: 1- quenching of hydroxyl radical(H0), 2inhibit $\mathrm{OH}$-dependent and $\mathrm{OH}$-induced peroxidation system, 4-inhibition of both non-enzymatic lipid peroxidation and lipo-oxygenase pathway. Thus, edaravone can improve ROS-induced neurological impairment in treatment of subarachnoid haemorrhage, acute ischemic stroke and intracerebral haemorrhage. ${ }^{26}$ In post ischemic inflammation state inhibit neutrophils activations as well as inducible NOS (i NOS) and neuronal NOS (nNOS) expression. The anti-inflammatory effects of edaravone have been confirmed in non-ischemic models of inflammation in extracerebral organs. Edaravone inhibit the oxidative modification of low-density lipoproteins (LDL), but also reverses oxidized LDL-induced reduction in expression of endothelial nitric oxide synthease (eNOS). ${ }^{27,28,29}$

\section{Membrane stabilizing effect:}

Edaravone present in the cell surface and/or near the surface of liposomal membrane thereby scavenges peroxyl radicals in both the aqueous and lipid phase. It suppresses the generation of ROS such as hydrogen peroxide and hydroxyl radicals of human neutrophils by its quenching action rather than by inhibiting neutrophils function. ${ }^{30}$

\section{Effects on non-neurological diseases (pleotropic effects):}

In addition to anti-stroke effects, edaravone prevent oxidative damages to various extracerebral diseases. Edaravone scavenges free radicals and has antiapoptotic, anti-cytokine effects in various diseases as it can diffuse into many disease affected organs and shows protective effects in the heart, lungs, intestine, liver, pancreas, kidney, bladder and testes. Thus, edaravone should be considered for treatment of diseases other than stroke focusing on its pleotropic effects. $^{31,32,33,34,35 .}$

\section{Anti-cytokine effects:}

Edaravone suppresses plasma monocyte chemoattractant protein-1(MCP-1), improve LV ejection fraction and reduce hospitalization due to heart failure with AMI. Decreases TNF- $\alpha$ production induced by I/R-induce injury, protect cardiac function and reduce infarct size in rats. In LPS-induced lung injury of mouse model, prevent lung injury and attenuates inflammatory cell and pro-inflammatory cytokines production such as IL$6, \mathrm{TNF}-\alpha$, kerationocyte derived chemokines and 
macrophage inflammatory protein-2 (MIP-2) in BALF. $^{36}$ Edaravone inhibit activation of phospholipase A2 and production of plateletactivating factors and ameliorates lung edema and leukocyte extravasation. ${ }^{24^{\prime} 32}$ After I/R-injury of rats liver edaravone suppress the expression of cytokines and chemokines such as TNF- $\alpha$, IL-1,IL- 8 , monocyte chemoattractant protein -2( $\mathrm{MCP}-2)$, macrophage inflammatory protein(MIP1 $\alpha, \mathrm{MIP}-1 \beta$ ) and reduces intracellular adhesion molecule1(ICAM-1) mRNA expression. Also suppresses endotoxin-induced liver damage by inhibiting expression of inflammatory cytokines and chemokines as well as inflammatory cell recruitment. Edaravone shows protective effects on ischemic insults and inflammation in heart, vessels and brain in experimental studies. ${ }^{37}$

\section{Effects on I/R-induced acute Pancreatitis (AP):}

Edaravone reduces pancreatic and intestinal injury after AP in mice. There was reduction in histological score, apoptosis, IL-6, IL-1 $\beta$ and TNF$\alpha$ along with obstructing activation of TLR-4 and NF-K $\beta$ activation.$^{38}$ It suppresses NF-k $\beta$ activation in LPS-induced lung injury in mice. ${ }^{39}$ TLR-4 can upregulate expression of inflammatory mediators via receiving ligand signal and protein molecules of necrotic cells and can enter extracellular fluid and recognized as endogenous ligands by TLR-4 to initiate immune response and sterile inflammatory response. Edaravone restrain TLR-4-NF-k $\beta$ pathway and repairs pancreatic and intestinal injury via regulation of TLR-4-NF-k $\beta$ signal pathway. ${ }^{40}$

\section{Effects on ischemic preconditioning of kidney:}

Edaravone significantly decreases serum creatinine and BUN concentration and ameliorates histological damage of renal tissue, decrease TUNEL-positive cells and Bax expression (marker of apoptosis). ROS are produced mainly by the tubular cells and cause lipid peroxidation, which is a free radical generating system closely related to I/R-induced tissue damage. Edaravone prevent depletion of SOD in the tubular cells and prevent lipid peroxidation, upregulate anti-apoptotic protein $\mathrm{Bcl}-2$ and downregulate pro-apoptotic protein Bax, thus prevent oxidative stress damage and apoptosis induced by I/R- injury. ${ }^{41}$

\section{Conclusions}

Edaravone a broad spectrum antioxidant scavenges ROS and RNS in both aqueous and lipid environments. Besides anti-stroke effects it has multiple pleotropic action includes antiinflammatory, anti-cytokine, immunomodulatory, anti-apoptotic, anti-necrotic, anti-fibrotic, membrane stabilizing, protect lung surfactant and protect multiple organs against I/R-induced injury. Thus, it appears to be most useful agent in prevention and treatment of COVID-19-induced cytokine storm, ALI/ARDS. However, prior to instituting antioxidant therapy, we must define the appropriate time points for intervention in each disease process. Antioxidant treatment become increasingly difficult as the inflammatory process and damage induced become irreversible with time.COVID-19 infection classically proceeds with initial phase of viral replication with high viral loads most likely benefit from effective antiviral drugs, where as the overlapping and third phase is manifested with hyperimmunity with high cytokines levels with low viral loads with aberrant immune response causing much of the host damage most likely benefit from anti-inflammatory drugs therapy i.e. steroid eg. Dexamethasone and anticytokine Tocilizumab etc. Edaravone started in the early phase and/or at the overlapping second phase may prevent progression to cytokine storm in high risk patients and ameliorates the manifestations of cytokine storm ARDS in third phase. It may decrease need for ventilation and/or duration of ventilation and mortality. Thus, it can be used as an adjunctive therapy for the treatment of severe COVID-19 infection. Effectiveness of edaravone is evident from treatment of Paraquat poisoning (a highly oxidative burst state caused by free radical damage leading to AKI,ARDS and/or multiorgan failure with similar pathophysiology of COVID-19induced cytokine storm ARDS), started within 24 hour of poisoning ,before development of complications had very low morbidity and 
mortality in mild to moderate poisoning. (unpublished recent personal observations in 7 cases of mild to moderate poisoning , did not developed any complications with early edaravone therapy in comparison to $>98 \%$ developed complications with high morbidity and mortality in retrospective observations treated with combinations antioxidants including IV N-Acetylcysteine and steroids etc). Thus, we need to explore the broad spectrum antioxidant edaravone alone or with combination of antioxidants for synergistic effects rather than a single agent. Large double blind placebo control dose finding study of early edaravone therapy should be considered in treatment of high risk COVID-19 patients to know whether edaravone prevent cytokine storm, ARDS and/or decreases the need for ventilation, duration of ventilation and mortality.

\section{Acknowledgement}

We are very much thankful to our family members in the coronavirus pandemic lockdown period for their kind cooperation and encouragement for this work for the benefits of COVID-19 patients. We have no any conflict of interest.

\section{References}

1. Serena Colafrancesco, Cristiano Alessandri, Roberta Prion et al.COVID-19 gone bad. A new character in the spectrum of the hyperferritinemic syndrome? Autoimmunity Review.2020, May 5.102573.

2. Reuter S, Gupta SC, Chaturvedi MM, Aggrawal BB. Oxidative stress, inflammation and cancer: how are they linked? Free Radical Biology and Medicine. 2010; 49:1603-1616. Doi. 10.1016/j. freeradbiomed. 2010.09.006.

3. Poyton RO, Ball KA, Castello PR. Mitochondrial generation of free radicals and hypoxic signaling. Trends in Endocrinology and metabolism.2009; 20(7):332-340. Doi.10.1016/j tem.2009.04.001.
4. Eileenn $\mathrm{M}$ Bulger, Ronald $\mathrm{V}$ Meir. Antioxidants in critical illness. Arch Surg.2001; 136:1201-1207.

5. Kalid A Hanaty, Magdy H Selim. Antioxidant strategies in neurocritical care. Neurotherapeutics.2012; 9:44-55. doi.10.1007/s13311-011-0085-6.

6. JD Capro. Oxidative stress as an initiator of cytokine release and cell damage. Eur Respir J. 2003; 22. Suppl. 44.4565 . Doi.10.1183/09031976.03.00000203a

7. Sahnoun Z, Jamoussi K, Zenghai KM .Free radicals and antioxidants: physiology, human pathology and therapeutic aspect. (Part II). Therape.1998;July-Aug.58 (4):315339.

8. Perricene C, Shoenfeld Y, Gerli R.COVID19 as part of hyperferritenemic syndrome. Implication for treatment. Autoimmun Rev.2020. (in press).

9. Sung Nim Han, Simin Nikbin Meydani. Antioxidants, Cytokines and influenza infection in aged Mice and Elderly Humans. Journal of Infectious Disease.2000; 182 (suppl):S74-80.

10. Meydani SN, Barklund MP, Liu S et al. Vitamin E supplementation enhances cellmediated immunity in elderly healthy subjects. Am J Cli Nutr.1990; 52; 557-63

11. Sun Y, Oberly LW. Redox regulation of transcriptional activators. Free Radic Biol Med.1996; 21:335-348.

12. Hennet T, Peterhans E, Stocker R. Alternation in antioxidants defense in lung and liver of mice infected with influenza $\mathrm{A}$ virus. J Gen Viraol.1992; 73:39-46.

13. Han SN, Meydani M, Wu D et al. Effects of long-term antioxidant supplementation in influenza infection. J Gerentol.2000. (In press).

14. Yang SN, Chen CS, Yiang GT, Cheng TL, Yong SB, Wu MY, Li CJ. New insight into the immune molecular regulation of the pathogenesis of acute respiratory distress 
syndrome. Int J Mol Sci. 2018; 19. doi.10.3390/ijms19020588.

15. Channappanavar R, Fehr AR, Vijoy R,Mack M, Zhao J, Meyorholz DK, Perlman J. Dysregulated type I interferon and inflammatory monocyte-macrophage response cause lethal pneumonia in SARinfected mice. Cell Host Microbe. 2016;19:181-193.

doi.10.1016/j.chem.2016.01.007.

16. Recalcati S, Invernizzi P, Arosio P,Cairo G. New functions for an iron storage protein: the role of ferritin in immunity and autoimmunity. J Autoimmun. 2008; 30:8489.

17. Ruddell RG, Hoang-Le D, Barwood JM, Rutherford PS, Piva JJ, Watters DJ. Ferritin function as a pro-inflammatory cytokine via iron-independent protein kinase $\mathrm{C}$ zeta/nuclear factor kappa $\beta$-regulated signaling in rat hepatic steallate cells. Hepatology. 2009; 49:887-900.

18. Dahan S, Katz I, Hellou T, Tietel M,Drob Y, Bry KG, et al. A fatal correlation: ferritin as a marker of severity in COVID-19 patients. Autoimmu Rev.2020 ;(in press).

19. Chung Wei Chow, Maria Teresa Herrera Abrece, Tomoko Suzuki, Gregory P Downey. Oxidative stress and Acute Lung Injury. Am J Respir Cell Mol Biol. 2003;29:p-427-431. doi.10.1165/ycmb. F278. www. atsjournals. org.

20. Mathew R Deshotel, Huijing Xia, Srinivas Sriramula, Eric Lazartigues, Catelin M Filipean. Angiotensin -II mediated Angiotensin converting enzyme type-2 internalization and degradation through an Angiotensin-II type 1 receptor dependent mechanism. Hypertension. 2014; 64: 13681375. https:// doi.10.1161/ HYPERTENSIONAHA 114.03743.

21. Shirin K Laleh, S Maryam BO,Ilianz R, Mine M, Mehrdad Z, Azahar S-jazi, Nagar K, Mohammad SD, Parisa S, Hosen K. The molecular story of COVID-19. NAD+ depletion addresses all questions in this infection. Preprints (www.priprint.org) posted on 23 March 2020. doi.10.20944/preprints 202003.0346.v1.

22. Martin Paspe Cruz. Edaravone (Radicava). A novel neuroprotective agent for the treatment of Amyotrophic lateral sclerosis.P.T.2018; 43(1):25-28.

23. Kazutoshi Wantanabe, Masahiko Tanaka, Satoshi Yuki, Manabu Hirai, Yorihiro Yamamoto.How is edaravone effective against acute ischemic stroke and amyotrophic lateral sclerosis? J Cli Biochem Nutr.2018 Jan; 62 (1): 20-38.Published online 2017 Nov 11.doi.10.3164/jcbn.17-62.

24. Reyes YA, Shimoyama J, Akamatsu H, Sunameri M. MCI-186 (edaravone) ,a free radical scavenger, attenuates ischemiareperfusion injury and activation of phospholipase $\mathrm{A}_{(2)}$, in an isolated rat lung model after $18 \mathrm{hr}$ of cold preservation. Eur J Cardiothoracic Surg.2006; 92:304-11.doi. 29304311200610.1016 ijcts.2005.12.0051642799.

25. Yun Yuan, Hao Zha, Parakalan Rangarajan, Eng-Ang Ling, Chupyun Wu. Antiinflammatory effects of Edaravone and scutellarin in activated microglia in experimentally induced ischemia injury in rats and in BV-2 microglia.BMC Neuroscience. 2014; $15: 125$. https://www.biomedcentral,com/13712202/15/125.

26. Jie Yang, Xiaoyang Cui,Jie Li,Canfei Zhang, Jing Zhang, Ming Lie. Edaravone for acute stroke: Metaanalysis of data from random and control trials. Developmental Neurorehabilitation. Early online 1-6. doi. 10.3109/ 17518423.2013.830153. https;//informahealthcare.com/pdx.

27. Kono H, Asakawa M, Fujii H, Maki A, Amemiya H, Yamamoto M, et al. (2003) Edaravone, a novel free radical scavenger, prevents liver injury and mortality in rats 
administered endotoxin. J Pharmacol Exp Ther 307:74-82.

28. Nomoto N (2004) Inhibitory effect of free radical scavenger, MCI-186, in the increase of hydroxyl radical induced by iminodipropionitrile in rats. J Neurol Sci 219:41-44.

29. Tanaka K, Takemoto T, Sugahara K, Okuda T, Mikuriya T, Takeno K, et al.(2005) Postexposure administration of edaravone attenuates noise-induced hearing loss. Eur J Pharmacol 522:116-121.

30. Makawa K, Akamatsu H, Nishina K,Obara $\mathrm{H}$, Niwa Y. Effects of edaravone on human neutrophil function. Acta Anaesthesiol Scand.2009;49:385-389.

31. Kiyoshi Kikuchi, Nobuyuki Takeshige, Naoki Miura, Yoko Morimato, Tkashi Ito, Salurya Tancharoen et al. Byond free radical scavenging: Beneficial effects of edaravone (Radicut) in various diseases with experimental and Therapeutic Medicine. Sept.20.2011; https://doi.org/10.3892/etm.20 11.352.p-3-8.

32. T Akaol Takeyoshi, O Totsuka, K Arakawa, M Muraoka, K Kabayashi, K Konn,K Matsumoto, Y Morishita. Effects of free radical scavenger MCI-186 on pulmonary ischemia-reperfusion injury in dogs. Heart Lung Transplant. 25965871200610.1016/j healn. 2006.03.00416890118.

33. W Qiu, H Gul, Zhang J, Zhou D, Chen Y, Chen et al. Pretreatment with edaravone reduces lung mitochondrial damage in an infant rabbit ischemia-reperfusion model. J Pediatr Surg. 4320532060200810.1016/jpedsurg. 2008.05.-01918970940.

34. S Tajima, M Bando, Y Ishii, T Hosono, $\mathrm{H}$ Yamasawa, S Ohno, T Takada, E Suzuki, F Gejyo, Y Sugiyama. Effect of edaravone, a free radical scavenger on bleomycininduced lung injury in mice. EUR Respir J. 3213371343200810.1183/09031936.001644 0718614556.
35. S Tajima, M Soda, M Bando, M Enomoto, H Yamasawa, S Ohno, T Takada, E Suzuki, F Gejyo, Y Sugiyama. Preventive effects of edaravone, a free radical scavenger, on lipopolysaccharide-induced lung injury in mice. Respirology. 13646653200810. 1111/j.1440-1843.2008.01322.x18713088.

36. $\mathrm{T}$ Asai, $\mathrm{Y}$ Ohno, $\mathrm{S}$ Minatoguchi, $\mathrm{N}$ Funaguchi, H Yuhgetsu,M Sawada, G Takemura, A Komada,T Fujiwara, H Fujiwara. The specific free radical scavenger edaravone suppresses bleomycin-induced acute pulmonary injury in rabbits. Clin Exp Pharmacol Physiol. 342226200710.1111/j.14401681.2007.04528.x17201731.

37. Kiyosi Kikuchi, Salunya T, Nobuyuki T, Munetaka T, Motohiro M, Yoshinaka M,Eiichiro T. The efficacy of edaravone (Radicut), a free radical scavenger for cardiovascular disease. Int $\mathrm{J}$ Mol Sci.2013;14:1390913930.doi.10.3390/ijms 140713909.

38. Bingbin Wang, Wendong Lin. Edaravone protect against pancreatic and intestinal injury after acute pancreatitis via nuclear factor- $\mathrm{k} \beta$ signaling in mice. Biol Pharm Bull.2020; 43(3):509-515.

39. Zhang Z, Luo Z, Bi A, Yang W, An W, Dong $\mathrm{X}$, Chen $\mathrm{R}$, Yang $\mathrm{S}$ et al. Compound edaravone alleviate lipopolysaccharide (LPS)-induced acute lung injury in mice. Eur J Pharmacol.2017; 811:1-11.

40. Zhang XP, Zhu CM, Wu DJ, Jing X. Possible role of Toll-like receptor-4 in acute pancreatitis. Pancreas. 2010; 39:819-824.

41. Li Yan,Xia A-Xhou, Xing Shu-hua. Protective effect of edaravone against renal ischemia/reperfusion injury and compared with ischemia postconditioning in rats. Acta Pharmaceutica Sinica.2010; 45(7):840-848. 